\title{
A framework for allocating personalized appliance-level disaggregated electricity consumption to daily activities
}

\author{
Simin Ahmadi-Karvigh ${ }^{\mathrm{a}}$, Burcin Becerik-Gerber ${ }^{\mathrm{b}}$, Lucio Soibelman $^{\mathrm{c}}$ \\ ${ }^{a}$ Research Assistant, Sonny Astani Dept. of Civil and Environmental Engineering, Univ. of Southern California, \\ KAP 217, 3620 South Vermont Ave., Los Angeles, CA 90089-2531. E-mail: ahmadika@usc.edu \\ ${ }^{\mathrm{b}}$ Associate Professor, Sonny Astani Dept. of Civil and Environmental Engineering, Univ. of Southern California, \\ KAP 224C, 3620 South Vermont Ave., Los Angeles, CA 90089-2531. E-mail: becerik@ usc.edu , Tel: +1 213740 \\ 4383 (Corresponding Author) \\ ${ }^{\mathrm{c}}$ Professor, Sonny Astani Dept. of Civil and Environmental Engineering, Univ. of Southern California, \\ KAP 210A, 3620 South Vermont Ave., Los Angeles, CA 90089-2531. E-mail: soibelman@usc.edu
}

\begin{abstract}
Residential and commercial buildings account for more than $74 \%$ of total annual electricity consumption in the United States. Studies have shown that occupants' awareness of their behaviors in consuming electricity encourages them to change their unsustainable behaviors and improves the sustainable ones. As behaviors impact the ways that daily activities are performed, in order to develop a personalized appliance level model of an occupant's behavior, precise activity recognition is required. In this paper, we introduce a novel framework to allocate personalized appliance-level disaggregated electricity consumption to daily activities. In our framework, using ontology based approach, the input appliance usage data is first separated into categories of non-overlapping activity events. The separated data sets are then segmented to detect activity segments, which are next mapped into activity classes using a trained classification model. To evaluate the performance of our presented framework, an experimental validation was carried out in three test bed apartment units. Results of validation showed a total F-measure value of 0.97 for segmentation and an average accuracy of $93.41 \%$ for activity recognition. Following the activity recognition, the approximate electricity consumption associated with the recognized activities was estimated and the results of each test bed unit were compared with the others.
\end{abstract}

Keywords: Energy awareness; Behavior-based consumption; Disaggregated electricity consumption; Daily activities; Activity recognition

\section{Introduction}

United States is the second largest electricity consumer in the world [1]. In 2014, residential and commercial buildings consumed more than $74 \%$ of the total electricity in the United States. Of this amount, about $60 \%$ was used for electric appliances/devices including lighting fixtures, kitchen appliances, computers, electronics and other household appliances/devices. An increasing amount of work supports the idea that awareness of personalized and detailed electricity consumption could assist occupants to reduce their consumption [2]. Along this line, various techniques have been successfully used to measure electricity consumption down to the device/appliance level [3-5]. With the aid of disaggregated electricity consumption data, occupants are able to distinguish inefficient appliances with anomalous electricity consumption and discover possible savings by substituting them with more efficient ones. However, the way these appliances are used by occupants also affects the total electricity consumption in buildings [6]. For example, Fechner showed that chefs, using the same equipment for cooking the same meal, had electricity consumptions with up to 50\% difference [7]. In another study, careless behavior have shown to increase a building's electricity consumption by one-third, while conservation behavior can save a third [8]. As behaviors impact the ways that occupants perform their daily activities, in order to achieve insight about occupant's behavior, exploration of activities is required. 
Daily activities are combinations of different actions performed by an occupant in order to satisfy a specific need. These actions and their durations are case sensitive. Even for a specific case, they could be inconsistent under different circumstances. For example, the activity of preparing breakfast can be formed by a variety of actions in different ways. Fig. 1 shows two possible behaviors in performing this activity.
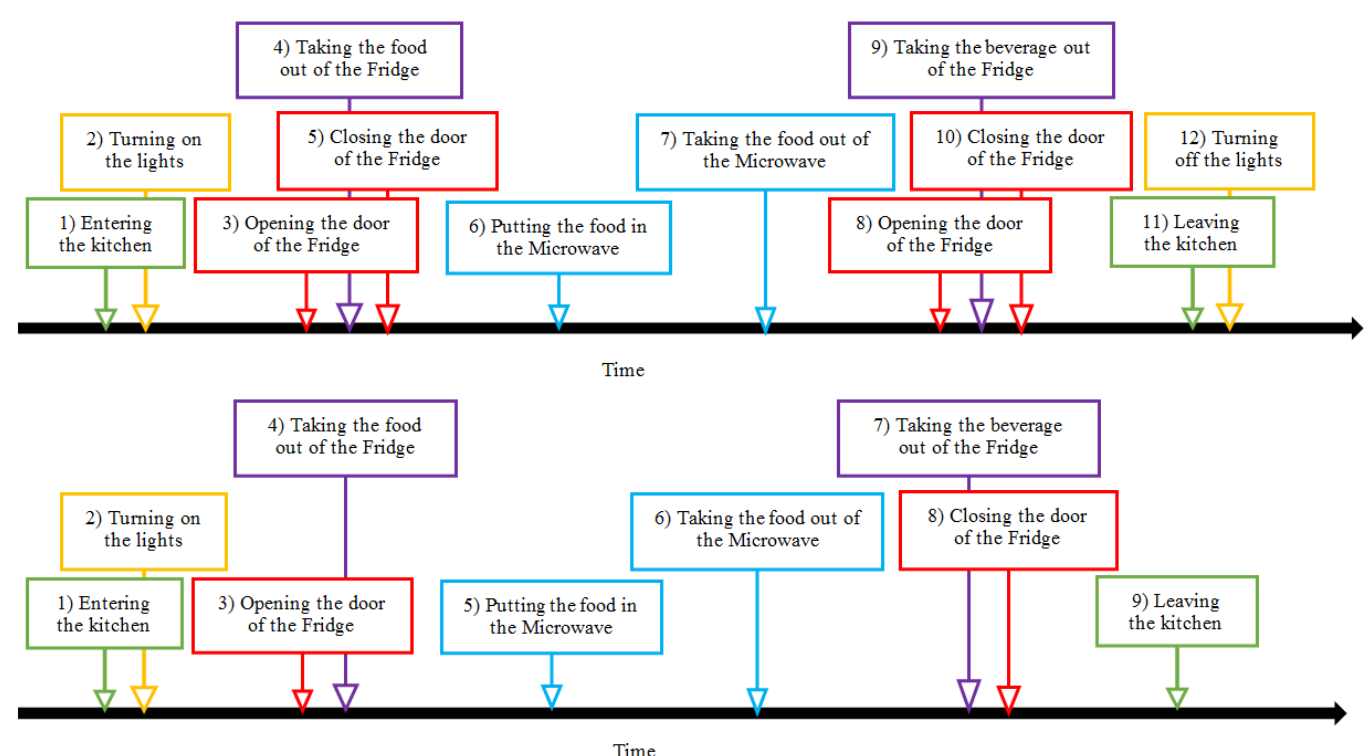

Fig. 1. Two sample behaviors in performing the activity of preparing breakfast. Reversed activities are shown by the same color (e.g., turning on lights and turning off lights)

Here, the slight difference in the associated actions by which the activities are formed causes more electricity consumption in the second behavior, as the fridge door is left open when the microwave is used and the lights are also left on after leaving the kitchen. As illustrated in this example, by recognizing and exploring activities, it is possible to develop an actual and personalized appliance level model of an occupant's behavior, based on which it is possible to detect potential energy savings and give feedback to occupants. Along this line, in this paper, we introduce a context-aware data-driven activity recognition framework using appliance-level disaggregated electricity usage. In contrast to the commonly used activity recognition approaches that require installation of multiple sensors in a building or require carrying wearable sensors by occupants, our approach relies solely on the data provided by a Non-intrusive Load Monitoring (NILM) system [9], using a single sensing point. The main contributions of this paper can be summarized as follows: (1) investigation of the application of the NILM technique in activity recognition by introducing a framework that recognizes occupant's daily activities using a single sensing point in the context of building energy efficiency; (2) introduction of a context-aware data separation approach to determine the possible overlapping activities via ontological reasoning (3) introduction of an unsupervised approach to detect the length of activities; and (4) real-world implementation of the framework in three residential units. Following the summary of related studies presented in the next section, the details of our proposed framework are provided in section three. The implementation of our framework in real-world test beds along with validation and discussion parts are presented in section four. Finally, limitations of the framework and future work are presented in section five and section six concludes the paper.

\section{Related prior work}

Researchers have been exploring activity recognition for a variety of applications over the past decade. One of the domains, in which activity recognition has been effectively explored, is the healthcare domain [10]. 
Supporting the idea of independent living, various studies have investigated activity detection approaches to monitor daily activities of elderly people in order to detect abnormal behaviors resulting from illnesses or emerging medical conditions and also to observe the progress of age-related diseases, such as Alzheimer [11-13]. In addition to daily activities, detection of physical movements has also been investigated for healthcare purposes, specifically to detect emergency situations via fall detection [14].

Another application domain of activity recognition is the energy management domain. Unlike the majority of the studies in the healthcare domain, where specific groups of people are investigated, i.e., patients and elderly people with limited level of activity due to their health conditions, there are usually higher variations and complexities associated with regular occupants' activities, which are to be detected for energy management purposes. Focusing on the energy management domain, there are studies that explored the influence of occupant's behavior on building's electricity consumption for smart grid [15-19]. In these studies, in order to create high-resolution energy demand models, historical time use and ownership data sets have been analyzed to create probabilistic models of activities and their associated electricity consumptions. Since these types of studies are based on large historical data sets, they do not reflect precise personalized consumption patterns. In another group of studies, activity recognition has been used for appliance standby mode or lighting system control [20-22]. Finally, there are a few studies that focus on possible applications of activity recognition for creating energy consumption awareness. For example, relying on smart meters, Chen et al. proposed an approach to map the electricity consumption to detected activities in smart homes [23]. Since in this study activities are first detected by a separate sensor network and then correlated to aggregated electricity readings from a smart meter, the approach lacked the disaggregated information of appliance usage for each activity. As compared to the healthcare domain, fewer studies exist in the energy management domain. Therefore, further investigation of activity recognition in the energy management domain, specifically for energy consumption awareness, is needed.

Regardless of the application domain, the techniques used by different activity recognition approaches could be classified into two main groups: data-driven approaches and knowledge-driven approaches [24]. In the data-driven approaches, machine-learning techniques and statistical methods have been adapted to detect activities using the data acquired by cameras or sensors installed in buildings. The majority of the existing approaches are supervised approaches and they require labeled data for training. There are also a few number of studies based on unsupervised techniques $[25,26]$, which generally suffer from lower performance comparing to the supervised approaches. Of supervised approaches, Hidden Markov Models (HMMs) [27, 28], Support Vector Machine (SVM) classifiers [29], Bayesian Networks (BNs) [30], Naive Bayes classifiers [31] and Decision Trees [31,32] are the most frequently used ones. In knowledge-driven approaches, activities are detected using ontological reasoning [33, 34]. Considering the contextual relationships, ontology-based approaches usually outperform data-driven approaches in detecting complex activities. However, they are usually not suitable for detecting simple and basic activities, as these activities can easily be detected with data driven approaches without the need of constructing an ontology, which is generally a time consuming process. For example, the social activity of giving a presentation to a group of participants in a conference room can be detected by ontology-based approaches. However, detecting single activities performed by a presenter or participants (e.g., entering the room or showing the slides) still relies on data-driven approaches.

Another feature that differentiates activity recognition approaches is the type of data used for detection. While earlier studies mainly explored approaches that relied on visual data provided by cameras [35, 36], privacy concerns associated with vision-based approaches moved the interest of researchers onto sensorbased activity detection approaches. A variety of sensors, including ambient sensors, motion sensors, RFID tags, smartphones and smart meters, have been used to detect daily activities [23, 29, 37, 38]. Since common approaches in activity recognition require the installation of multiple sensors to recognize activities, the high complexity and cost of installation are known drawbacks of these approaches. In order to address this issue, Berenguer et al. proposed an activity detection approach based on single sensing point on main power 
line with the purpose of supporting independent living of elderly people in the health care domain [13]. In

this approach, predefined weights have been used to correlate the activities with appliances. However these types of assumptions work well only for occupants, such as elderly people or patients, who have daily patterns with small variations from a predefined baseline.

In this paper, we explore activity recognition by introducing a novel framework, in which the daily activities are detected via our data-driven activity detection approach, using the data provided by a NILM system. The purpose of our framework is to estimate the personalized appliance usage for different daily activities performed by regular occupants in a building. In addition to providing the appliance level electricity consumption of daily activities, our framework eliminates the need of separate sensor network for activity detection.

\section{Appliance-level disaggregated electricity consumption for daily activities}

Our framework for appliance-level disaggregated electricity consumption for daily activities has three main parts: Context-aware data separation; Segmentation and Activity recognition; and Electricity consumption estimation (Fig. 2). As an input, this framework receives the labeled data of appliance usage, provided by an NILM system. The input data is first separated based on context information using ontological reasoning. The separated data is then segmented in order to detect the lengths of activities and generate the feature vectors, by which the activities are detected via a classification model. Finally, the contribution of appliances in electricity consumption of the recognized activities is estimated. The mentioned parts are extensively explained in the following sub-sections.

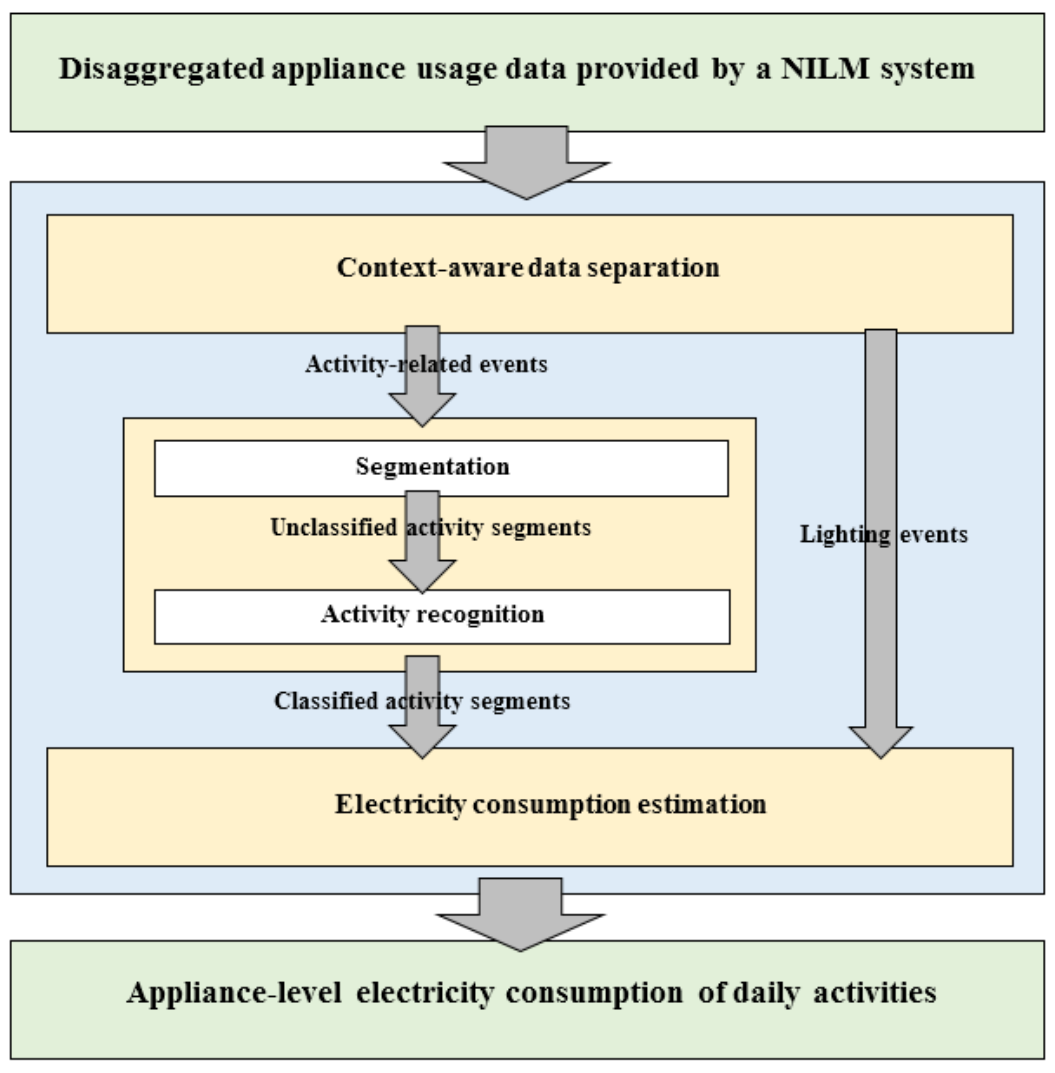

Fig. 2. Components of the framework 


\subsection{Input to framework}

The input to our framework is disaggregated appliance usage data provided by an event-based NILM system that uses a single sensing point on main power line. Electricity disaggregation using NILM technique was first introduced by Hart almost 30 years ago [9]. This technique relies on the idea that changes in the state of appliances (on or off), known as events, generate distinctive signatures in the power signal. In order to detect these signatures we used a Generalized Likelihood Ratio Test event detector, which is a probabilistic event detection algorithm [39, 40]. Fig. 3 shows some examples of the events generated by different appliances on the power signal. The differences in the amplitude and shape of the signatures, suggest that using a previously trained classifier, it is possible to map the detected events onto the appliances. As it is common in all classification problems, performance of the classifier is highly related to the features that are used for classification. Studies have shown that the extracted features from power signal depend on the resolution of the signal. The higher the signal resolution, the more information can be obtained from the extracted features. Therefore, in this study, in order to acquire high-resolution signal measurements, we used a high-frequency data acquisition system, consisting of voltage and current sensors installed on the main AC power line. The main components of AC power flow are: real power, reactive power and apparent power. Real power represents the capacity of the circuit and it is consumed thoroughly in the load. However, reactive power, which is generated when there is a phase difference between current and voltage waveforms, is first stored in the load and then returned to the grid. The vector sum of real and reactive powers is called apparent power. Among these three components, i.e., real power, reactive power and apparent power, real and reactive powers were used as features for classification. In order to extract these features, the acquired current and voltage measurements were first processed using mathematical transforms. Along this line, based on an approach presented in [41], we applied Short-time Fourier transform on current and voltage to obtain the transformed vectors ( $I$ and $V$, respectively). The presence of non-linear loads in a power system causes harmonic frequencies that must be taken into account in real and reactive power computations. Using the transformed vectors of $I$ and $V$ the real and reactive power components for different harmonics were computed via the following equations [42]:

$P_{k}(t)=\left|I_{k}(t)\right| \cdot \sin (\theta(t)) \cdot\left|V_{1}(t)\right|$

$Q_{k}(t)=\left|I_{k}(t)\right| \cdot \cos (\theta(t)) \cdot\left|V_{1}(t)\right|$

In these equations, $k$ represents the harmonic index and can get values from 1 to $9 . P_{k}, Q_{k}$ and $I_{k}$ are, respectively, real power, reactive power and transformed current waveform for the kth harmonic. $V_{1}$ represents the normalized negative-frequency coefficient of the transformed voltage waveform for the first harmonic. Finally, $\theta$ is the angle of $V_{1}$, which represents the voltage phase shift relative to the Fourier Transforms window.

For each detected event, the computed real and reactive powers for different harmonics via equations (1) and (2), in a time series segment with fixed length that contains the event, forms the feature vector $X_{n}$ [42]:

$X_{n}=\left\{P_{1}[n], Q_{1}[n], \ldots, P_{k}[n], Q_{k}[n]\right\}$

Obtained feature vectors of detected events were then classified into appliance classes using a classifier that was previously trained with the NILM labeled data. For each classified event $i$, a vector $(E)$ carrying the label $(l)$, time of occurrence $(t)$ and change in first harmonic real power $(\Delta P)$ associated with the event was stored in a local database $\left(D B_{\text {Input }}\right)$, which was later used as an input for the rest of framework:

$E_{i}=\left\{l_{i}, t_{i}, \Delta P_{i}\right\}$

$D B_{\text {Input }}=\left\{E_{i}\right\}$ 


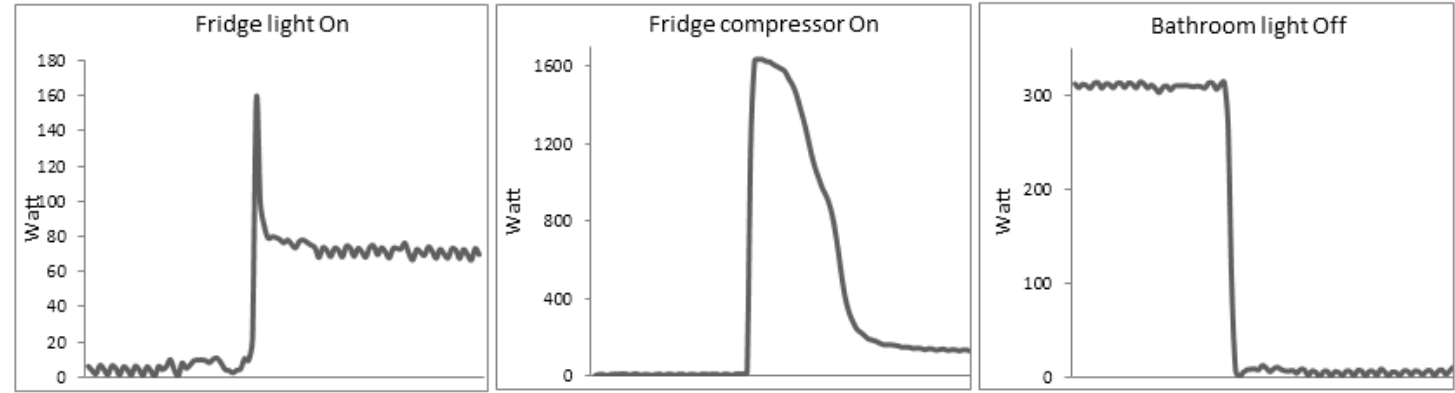

Fig. 3. Example of events generated by different appliances on real power signals

\subsection{Context-aware data separation}

Appliances are electrical devices that perform specific jobs in houses. In this paper, we classify appliances into three main categories: background appliances, lighting appliances, and activity-related appliances. Those appliances that are not directly associated with daily activities are classified as background appliances (e.g., an HVAC system, water heater and fridge). As the operation of these appliances is function of various factors, it is not possible to directly allocate their energy consumption to occupant's daily activities. Therefore, background appliances are not included in the focus of this study and accordingly the data of their events is eliminated from the rest of the input data. Once the data of background appliances is eliminated, the remaining data is either associated with lighting appliances (e.g., lighting fixtures), which can be used both during a daily activity or not, or with the appliances that are always used during an activity (e.g., a microwave), and we categorize them as activity-related appliances. Since the events associated with activity-related appliances typically occur during an activity, they are appropriate indicators of daily activities. Hence, the events of activity-related appliances are used for activity recognition. However, for energy estimation of detected activities, in order to consider the contribution of lighting appliances in energy consumption of the activity, the lighting appliances are also taken into account in addition to activity-related appliances.

In addition to the mentioned data separation of activity-related appliances from lighting appliances, in order to detect overlapping activities, i.e., activities that are performed in overlapping time intervals, further event separation of activity-related appliances based on potential overlapping activities is carried out. For brevity, herein after, by appliances and events, we mean activity-related appliances and the events associated with them. Although manual identification of potential overlapping activities in simple cases with small number of activities is possible, we propose an automated approach to support both scalability and adaptability, using Web Ontology Language (OWL). In order to identify whether there is a possibility for given activities to be performed in overlapping time intervals, additional context information is required. For instance, an occupant is preparing breakfast, using toaster and coffeemaker, while listening to music on the radio, which is also located in the kitchen. In this example two activities of preparing breakfast and listening to music can be performed simultaneously as they require uncommon appliances that are located in the same space. However, being associated with different appliances does not necessarily mean that two activities are overlapped. For example, although one might use coffeemaker only for preparing breakfast and oven only for preparing dinner, these two activities cannot be overlapped as they are habitually always performed in non-overlapping time intervals of the day. Accordingly we use time, space and appliances as indicators of context and identify the overlapping activities based on the modeled context indicators. Along this line, we use ontology to formally model activities and stated context indicators as concepts that are connected with predefined taxonomic and non-taxonomic relationships. Fig. 4 illustrates the created ontology. Such ontology contains classes (shown by ovals) as sets of individuals with common characteristics and properties (shown by arrows) as relationships between individuals of different classes. 


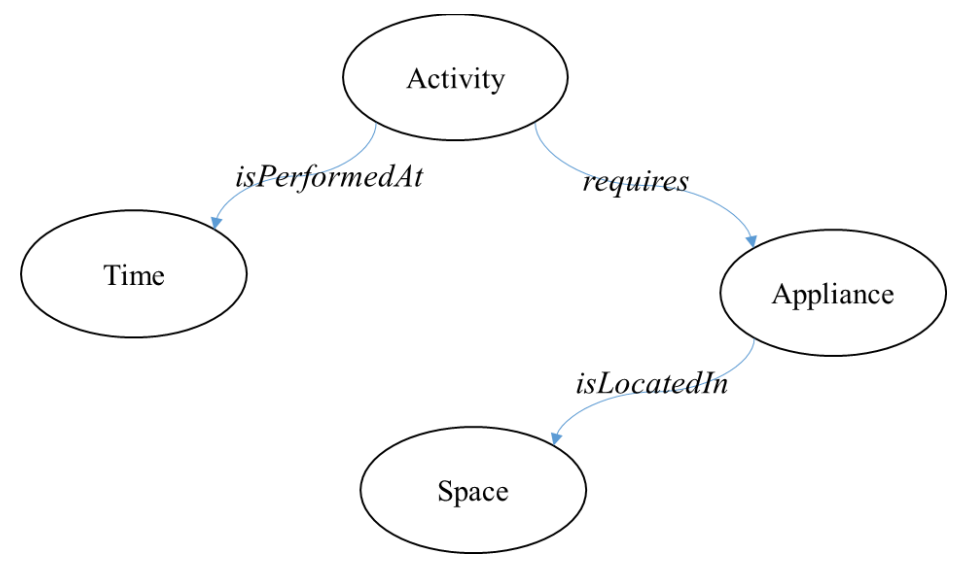

Fig. 4. Schematic view of the ontology with its main classes and properties

In order to model the ontology, we used OWL, which is based on a family of knowledge representation language called descriptive logic (DL) [43]. OWL makes it possible to model the semantics of concepts in a well-structured format, i.e., DL knowledge base, and derive mathematically proven facts using deductive reasoning. Two main components of DL knowledge base are TBox and ABox. TBox, which is the terminological component of the knowledgebase, holds the descriptive statements about the classes and properties. On the other hand, ABox, which is the assertional part of knowledgebase, is related to statements about individuals. For example, "Activity of preparing breakfast requires coffee maker or toaster, which are located in the kitchen" is a sample statement of TBox, while "Occupant's current location is kitchen and he is using coffee maker and toaster to prepare breakfast" is a sample ABox statement. Other than general definitions of classes and properties, in order to attain an adequate representative model, based on which accurate reasoning can be carried out, additional TBox assertions must be added to the knowledge base as explained below:

Subsumption: Subsumption supports the subclass-superclass relationship. Subsumption states that all members of a class are also members of their superclass. All main classes of our ontology have subclasses. For instance, activity of watching television (WatchingTV) is subclasses of class activity (Activity) and living room (LivingRoom) is subclass of class space (Space). Since all classes are subclass of class Thing in minimum level, watchingTv is also member of class Thing, as its superclass (Activity) is subclass of class Thing.

Disjointness: OWL is a language with open world assumption. Based on this assumption, a statement cannot be false unless it is unconditionally proven to be false. For example, being a member of a class does not falsify the membership of another class. Accordingly, a given occupant's current location can be both member of class Kitchen and class Bedroom, which is a contradiction in reality. In order to address the explained issue, we use Disjointness assertion. Disjointness assertion states that two disjoint classes do not have any common member. Considering open world assumption, all defined classes may have common members unless their disjointness is asserted in the knowledge base.

Class Restriction and Union: Restrictions on classes illustrates whether an individual is eligible for class membership. We use these restrictions for subclasses of class Activity but it can be further extended to other classes as well. For example, instances of class PreparingBreakfast needs at least one appliance from instances of class Coffeemaker or class Toaster. Here is where we need to use assertion of union, as several activities are associated with more than one appliance and not necessarily require all of them. In previous example of preparing breakfast, one might use only coffeemaker one day and the other day both coffeemaker and toaster. In either case, as long as the restriction of using at least one 
appliance from the stated group is satisfied, the reasoner concludes that activity of preparing breakfast is 256 performed.

257 As explained, assertions of class and property definitions along with some additional assertions form the 258 TBox component of the knowledgebase. In order to identify the overlapping activities, we propose the 259 algorithm shown in Fig. 5 flowchart. In this algorithm, the possibility of each two activities being occurred 260 at the same time interval is investigated, by adding statements into ABox and check whether the added 261 information (ABox) complies with previous assertions in TBox. The algorithm receives the knowledgebase $262(\mathrm{~KB})$ and the arrays of A, App and S, representing the set of all subclasses in main classes of Activity, 263 Appliance and Space, respectively, and constructs an empty array for output, called Overlapping. For 264 all possible 2-permutations of activities, appliances and spaces, the algorithm adds assertions for individuals 265 to ABox. Since for each activity subclass, we model a particular time subclass with defined naming format 266 that is predictable from the name of activity subclass (e.g., for class of PreparingBreakfast we have 267 PreparingBreakfastTime), a separate loop for time subclasses is not considered and the algorithm 268 adds the time assertions with regards to activity assertions. Following the modification of ABox, the 269 reasoner runs a consistency check for current condition of the knowledgebase. If the knowledgebase is consistent, it can be concluded that the asserted two activities and consequently the events associated with the related appliances can be overlapped. The algorithm adds these appliances into Overlapping array, which will be later used as basis for data separation. For next iteration, the added assertions are removed 273 from the knowledge base. 


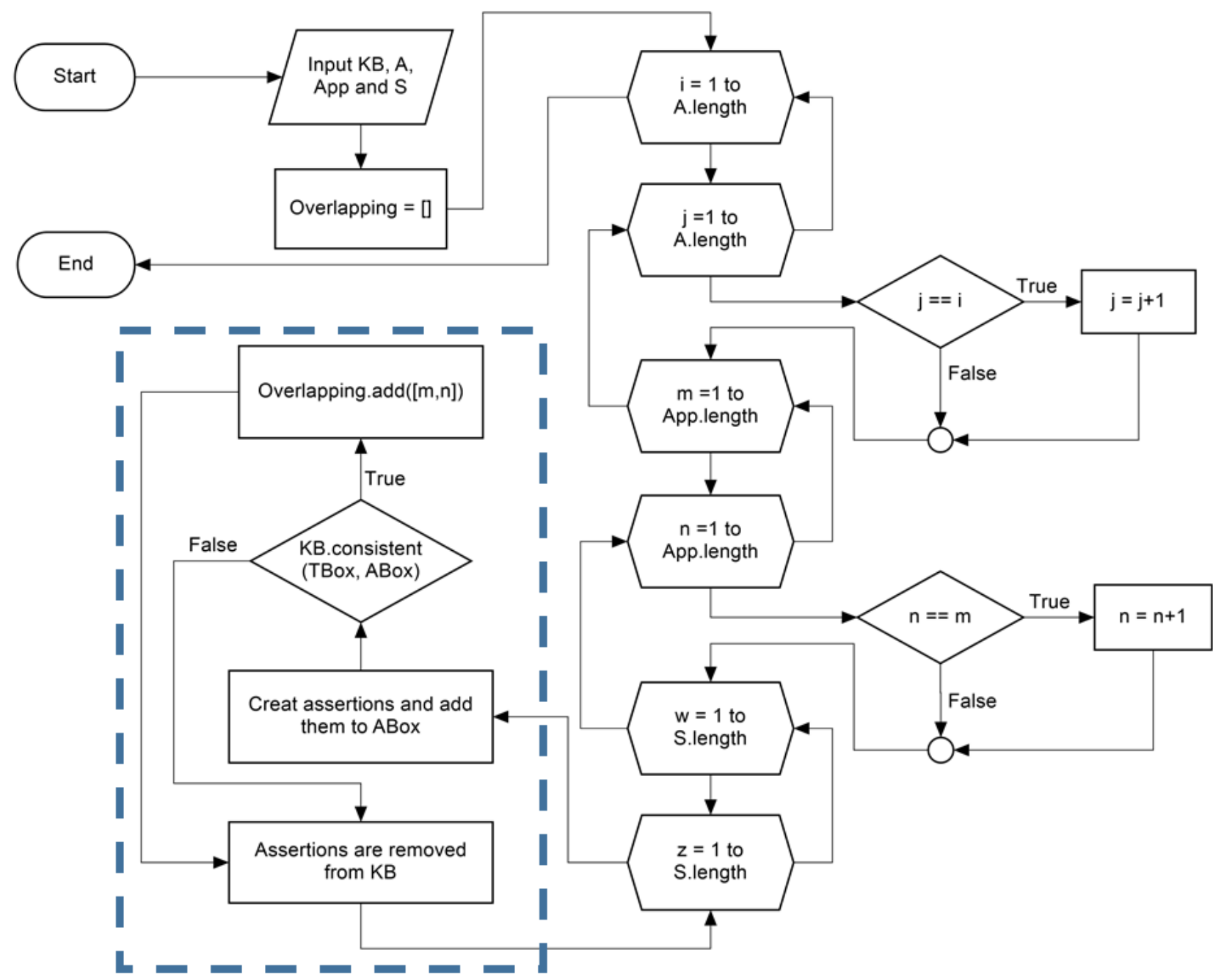

Fig. 5. Flowchart of the algorithm to identify whether two activities can be overlapped or not. The box shows the core part of the algorithm. KB is the knowledge base, which consists of TBox and ABox. A,

App and S represent main classes of Activity, Appliance and Space, respectively. The output of the algorithm, i.e., Overlapping, is an array that holds pairs of numbers. Each pair represents the indices of two appliances that are associated with possible overlapping activities.

The following example better illustrates the explained procedure:

"We are studying 4 activities of a female occupant, who lives in a one-bedroom apartment. The daily activities are: preparing breakfast, preparing dinner, watching television, and grooming. According to the occupant's lifestyle, she always prepares breakfast and takes a shower before leaving home to work and comes back at night and prepares her dinner. For preparing breakfast, the occupant uses electrical appliances including coffeemaker, oven and toaster, but not always all of them. For preparing dinner, the occupant uses the same appliances that are used for preparing breakfast, except the coffeemaker. In the morning following taking a shower, the occupant spends more time in the bathroom using the hair dryer or iron for grooming. In addition to these activities, this occupant watches television throughout the time she is at home. Although the television and the DVD player are located in the living room, since there is a view access from her open kitchen to the television in the living room, the occupant sometimes watches television while preparing a meal. “ 
The above scenario is human understandable and accordingly it is a trivial task for human to identify whether two activities can be overlapped or not. However, in more complicated situations, where more variations in activities, appliances and occupants exist, we need to rely on a systematic approach to avoid potential human errors and misconceptions. Along this line, we converted the stated scenario into a machine understandable format:

We are investigating 4 activities; therefore, we define 4 subclasses in class Activity and consequently 4 subclasses in class Time, representing the time in which each activity is performed. Based on these activities, we model 7 subclasses in Appliance class and three subclasses in class Space in order to represent the location of these appliances. Table 1 summarizes the names of the explained classes.

\begin{tabular}{|l|l|l|l|l|l|}
\hline Main Class & \multicolumn{2}{|c|}{ Subclass } & Main Class & \multicolumn{2}{c|}{ Subclass } \\
\hline Activity & PreparingBreakfast & A[1] & Space & LivingRoom & S[2] \\
\hline Activity & PreparingDinner & A[2] & Space & Bathroom & S[3] \\
\hline Activity & WatchingTV & A[3] & Appliance & CoffeMaker & App[1] \\
\hline Activity & Grooming & A[4] & Appliance & Toaster & App[2] \\
\hline Time & PreparingBreakfastTime & T[1] & Appliance & Oven & App [3] \\
\hline Time & PreparingDinnerTime & T[2] & Appliance & TV & App[4] \\
\hline Time & WatchingTVTime & T[3] & Appliance & DVDPlayer & App[5] \\
\hline Time & GroomingTime & T[4] & Appliance & HairDryer & App[6] \\
\hline Space & Kitchen & S[1] & Appliance & HairIron & App[7] \\
\hline
\end{tabular}

Finally to model the relationships between these classes we define 3 properties, i.e., isPerformedAt, requires and isLocatedIn. Fig. 6 shows how these properties relate the instances of different subclasses. As shown in this graph, all subclasses are disjointed except T[1] and T[3], to model the fact that activities of preparing breakfast and watching television can be performed at the same time; T[2] and T[3], to model the fact that activities of preparing dinner and watching television can be performed at the same time; and finally S[1] and S[2], to model the fact that the open kitchen space is not completely isolated from the living room.

These assertions create the TBox component of the knowledgebase, but the ABox is still empty. To tests whether two particular activities can be overlapped according to the modeled scenario, we need ABox assertions as follows:

Assertions for individuals:

Assertion1: A[i](sample-activity1), Assertion2: A[j](sample-activity2)

315 Assertion3: T[i](sample-time), Assertion4: T[j](sample-time)

316 Assertion5: App [m](sample-appliance1), Assertion6: App[n](sample-appliance2)

317 Assertion7: S[w](sample-location), Assertion8: S[z](sample-location)

Assertions for properties: 
Assertion 12: A[j](sample-activity2).requires $(P[n]($ sample-appliance 2$)$

Accordingly, in each iteration, the algorithm adds two assumed instances of activities (sample-activityl and sample-activity2) to the $i$ th and $j$ th activity subclasses $(A[i]$ and $A[j]$ ); an assumed instance of time (sampletime), which is the member of both the $i$ th and jth time subclasses (T[i] and $T[j])$; two assumed instances of appliances (sample-appliancel and sample-appliance 2) to the $m$ th and $n$th appliance subclasses (App [m] and App[n]); and finally an assumed instance of location (sample-location), which is a member of both the $w$ th and $z$ th space subclasses $(S[w]$ and $S[z])$. In order to relate the defined instances, the algorithm also adds assertions for properties in ABox. These assertions denote that sample-activityl and sample-activity 2 are both performed at sample-time. The former activity requires sample-appliance 1, while the latter requires sample-appliance 2 . Both sample-appliancel and sample-appliance 2 are located in samplelocation. Following the addition of these statements, in order to check whether the newly added statements are not in contradiction with the permanent TBox statements, the consistency of the knowledgebase is tested. In case the knowledgebase is consistent, which means one can perform the $i$ th and $j$ th activities at the same time using the $m$ th and $n$th appliances, the pair of $(m, n)$ is stored in output array (Overlapping). Since $m$ th and $n$th appliances are associated with possible overlapping activities, the events related to these appliances must be separated for activity recognition. The stated procedure iterates until all possible cases are tested. In this example, the output of the algorithm will be:

$\{(1,4),(2,4),(3,4),(1,5),(2,5),(3,5)\}$

which implies that the events associated with App[4] and App[5] must be separated from the events associated with App[1], App[2] and App[3]. Accordingly the events must be separated into different sets. First set contains the events associated with App[1], App[2] and App[3] and the other one contains the events associated with App[4] and App[5]. Other events, i.e., events associated with App[6] and App[7], can be included in first set, the second set or a new set of their own. 


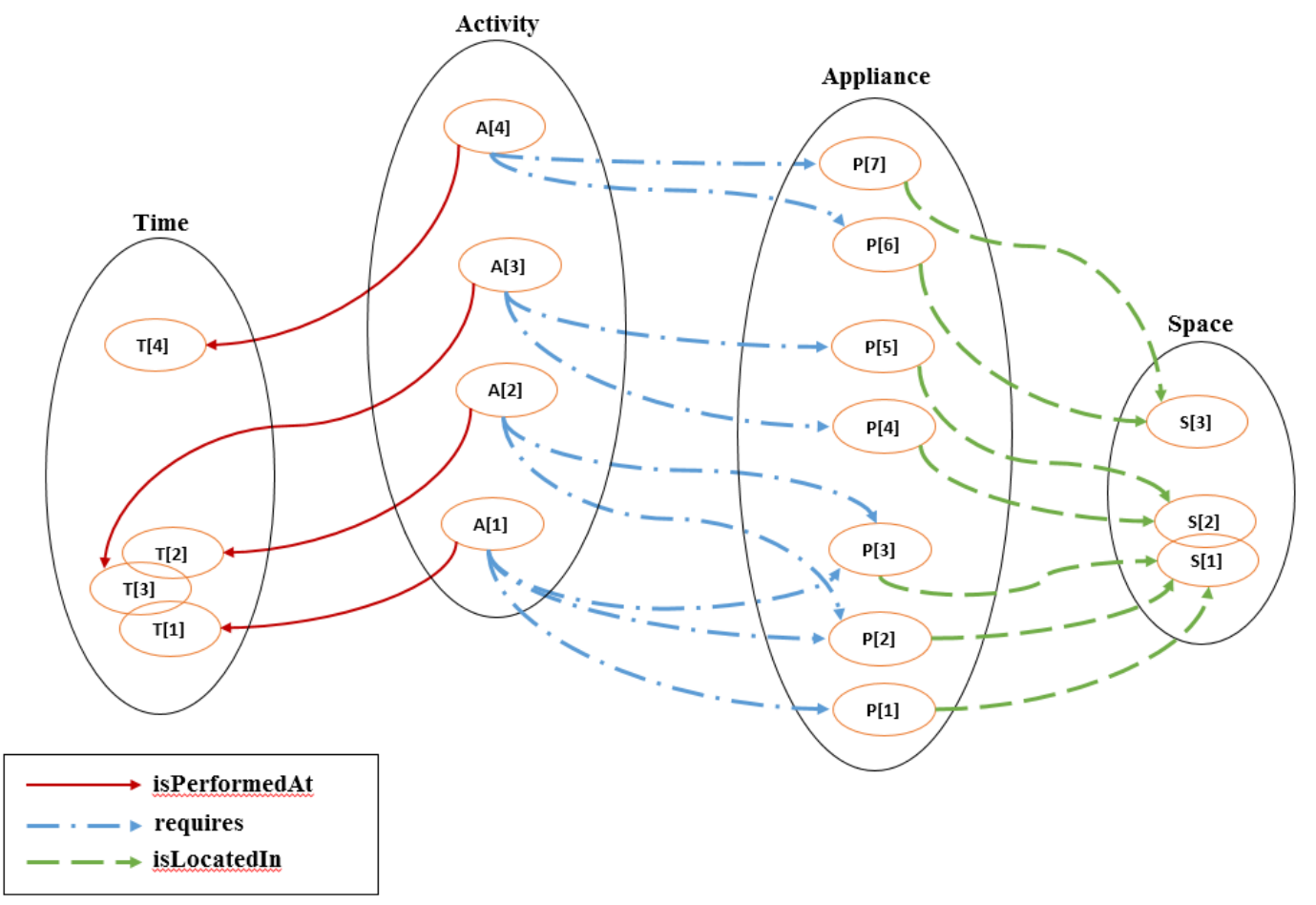

Fig. 6. Schematic view of the ontology in the example scenario

\subsection{Segmentation and activity recognition}

\subsubsection{Segmentation}

The datasets generated from the context-aware data separation step, i.e., activity-related appliance events associated with non-overlapping activities, are segmented into active and inactive intervals through an unsupervised segmentation process. Active intervals are those durations, in which the occupant is performing an activity and therefore, events affiliated with appliances occur. In contrast, during inactive intervals, due to the inactivity of the occupant, there are no events. Since in this paper, we are investigating activities that are associated with appliances, we assume that the minimum number of events in an active segment is two, i.e., one for appliance state change to "on" and the other to "off". For example, a segment associated with watching television might contain only one "on" and one "off" event for television. On the other hand, being associated with several appliances or multiple uses of an appliance, an activity might hold more than two events. For example, a segment representing the activity of preparing breakfast might contain several "on" and "off" events for using microwave, toaster and coffeemaker.

Given that the datasets are analyzed on a 24-hour basis, in order to detect the active segments, we used the heuristic that the lengths of activities and consequently the time differences between an activity's associated events are significantly smaller than inactive intervals between the activities. In other words, considering the events in a day, the activities are associated with segments that have relatively dense events. Hence, by estimating the density function of occurrence time of events, it is possible to detect the activity segments bounded by inactive intervals with zero density. For this purpose, the density functions are first estimated using the Kernel Density Estimation (KDE) with Gaussian kernel function via Eq. (6) [44].

$$
f(x)_{h}=\frac{1}{n h} \sum_{i=1}^{n} K\left(\frac{x-x_{i}}{h}\right)
$$


where $f$ is the estimated density function of a sample $\left(x_{1}, x_{2}, \ldots, x_{n}\right), K$ is the kernel function and $h$ is the smoothing parameter called the bandwidth. Among the available kernel functions, we used the Gaussian kernel function, which is the most commonly used. We calculate the optimum value of the bandwidth via an approach based on minimizing the expected loss function, proposed in [45]. Fig. 7a visualizes the events, occurred in a sample kitchen during a 24-hour day, in a chronological order. Fig. $7 \mathrm{~b}$ demonstrates how active and inactive segments of the dataset in Fig. 7a could be detected via the KDE.

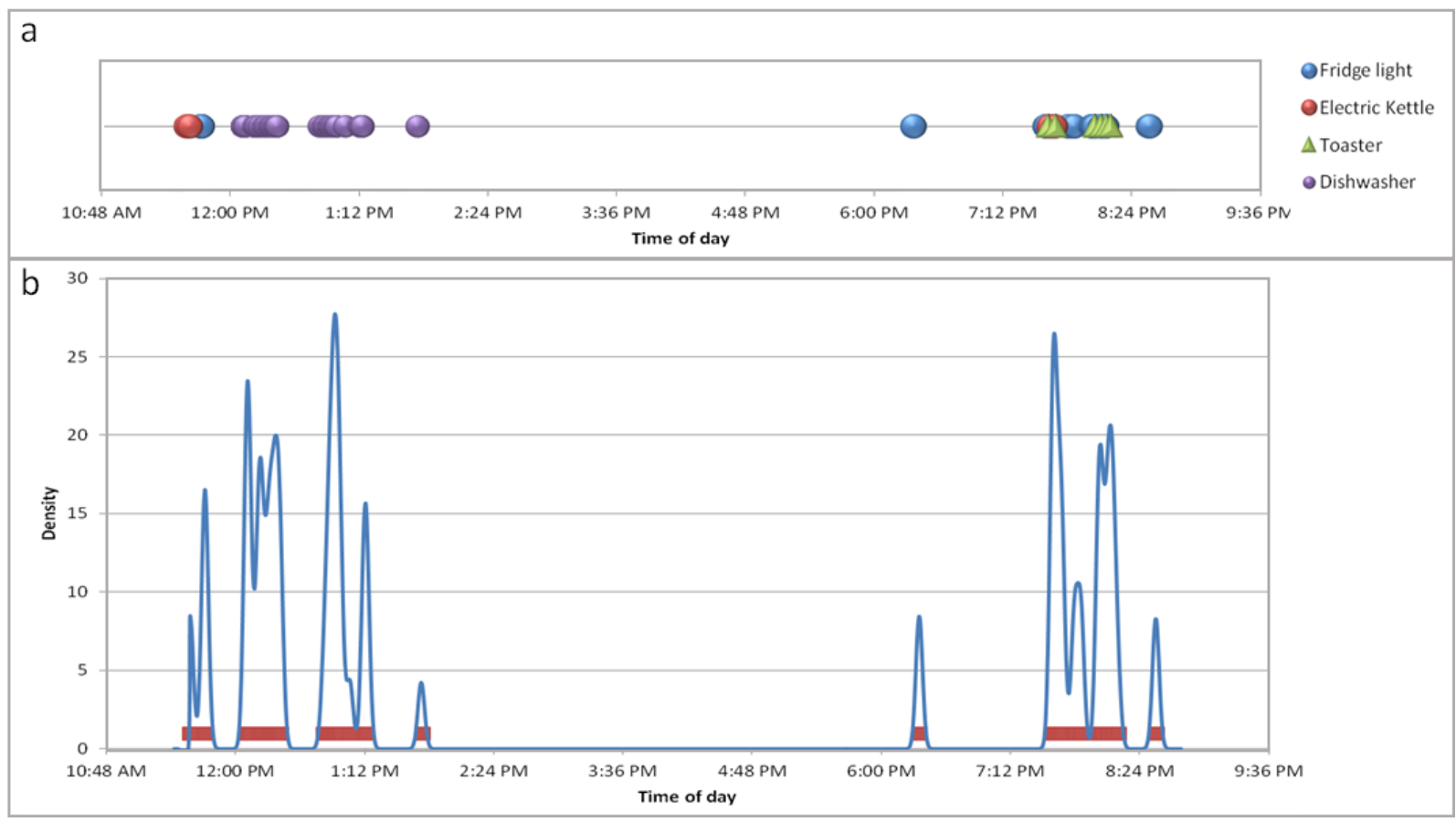

Fig. 7. Example of event segmentation; (a) chronological order of kitchen events occurred during a sample day; (b) Segmentation of the events shown in figure (a). Horizontal red lines illustrate the active segments.

\subsubsection{Activity recognition}

As explained in section 3.3.1, an active segment holding $m$ events starts and ends with events at time $t_{i}$ and $t_{i+m}$, respectively. Following the segmentation process, feature vectors $(F V)$ of the active segments $(\mathrm{Seg})$ are computed. The feature vectors consist of two parts. Each component in the first part corresponds to an appliance and carries the total duration, in which the appliance is used within a segment $\left(\Delta t_{A p p_{j}}\right)$, and the second part is the start time of the segment:

$\operatorname{Seg}_{t_{i}-t_{i+m}}=\left\{E_{i}, E_{i+1}, \ldots, E_{i+m}\right\}$

$F V_{t_{i}-t_{i+m}}=\left\{\Delta t_{A p p_{1}}, \Delta t_{A p p_{2}}, \ldots, \Delta t_{A p p_{n}}, t_{i}\right\}$

In order to map the segments to daily activities, a classification algorithm is required. Since classification is a supervised procedure, for a given set of daily activities, obtained labeled instances, i.e., feature vectors whose class label are known, are initially used to train the classification algorithm. Following the training process, the trained classifier is used to classify the new instances, i.e., feature vectors whose class label are not known, into activity classes. More details regarding the training and selection of classification algorithm are provided in section 4. 


\subsection{Electricity consumption estimation}

Following the activity recognition, the framework estimates the electricity consumption of detected activities via multiplying the power consumption of appliances by the duration of appliance usage. As depicted in Fig. 2, this part of the framework receives two inputs: classified activity segments and the lighting dataset containing the data related to lighting events. In order to determine the approximate contribution of each activity-related appliance in electricity consumption associated with an activity, for each segment, the energy consumption of an appliance usage is computed via the following equations:

$$
\begin{aligned}
& \left(P_{\text {average }}\right)_{u_{i}}=\frac{\left|\left(\Delta P_{\text {on }}\right)_{u_{i}}\right|+\left|\left(\Delta P_{o f f}\right)_{u_{i}}\right|}{2} \\
& (\Delta \mathrm{t})_{\mathrm{u}_{\mathrm{i}}}=\left(\mathrm{t}_{\mathrm{off}}\right)_{\mathrm{u}_{\mathrm{i}}}-\left(\mathrm{t}_{\mathrm{on}}\right)_{\mathrm{u}_{\mathrm{i}}} \\
& (E C)_{A p p_{j}}=\sum_{i=1}^{n}\left[(\Delta t)_{u_{i}} \times\left(P_{\text {average }}\right)_{u_{i}}\right]
\end{aligned}
$$

Where $u_{i}$ is the $i$ th usage of an appliance within the activity segment, $\left(\Delta P_{o n}\right)_{u_{i}}$ and $\left(\Delta P_{o f f}\right)_{u_{i}}$ are, respectively, the power changes associated with on and off events of $u_{i},\left(t_{o n}\right)_{u_{i}}$ and $\left(t_{o f f}\right)_{u_{i}}$ are the start and end times of the activity segment, and finally $(E C)_{A p p_{j}}$ is the estimated energy consumption of the appliance during that activity. As shown in these equations, we assume that the power consumption of the appliance in each usage is constant and equal to the average of power changes in on and off events associated with that usage. Multiplying the calculated average power consumption by the duration of appliance usage gives the energy consumption of the appliance during that operation. The total energy consumption of the appliance during the activity is achieved by summing over the energy consumption of all operations of that appliance in that activity segment. Although this approach is not precise, it gives an acceptable approximation of energy consumption for those appliances whose power consumption is almost constant or linear during their different modes of operation or cycle, which is also the case for the majority of activity-related appliances. To compute the contribution of lighting appliances in electricity consumption of the activity, the length of the activity, i.e., the length of the activity segment, is multiplied by the power value of the turned on lights that are located in the space that activity is performed.

\section{Validation results}

In order to evaluate the performance of our presented framework, we carried out experimental validation in three single occupancy test bed apartment units. The details of these experiments are presented in the following sub-sections.

\subsection{Test beds and data collection}

The presented framework has been implemented in three test bed apartment units. Of these three test beds, two of them were one-bedroom units with 4 separable spaces including kitchen, bathroom, living room and bedroom; and the third one was a studio, with 3 separable spaces including kitchen, bathroom and living room. All units were single occupancy units and they were located in the city of Los Angeles. The data acquisition was carried out for 11, 13 and 16 days in the three units. The occupants were asked to continue their regular activities during the experiments. The test beds were equipped with our prototype NILM system, monitoring the main power line at the electricity panel available inside the apartments. To do so, voltage and current sensors, i.e., Pico TA041-25 MHz $\pm 700 \mathrm{~V}$ differential probe and Fluke i200 AC current clamps, were installed on the main circuit breaker in each apartment for high frequency sampling $(1 \mathrm{kHz})$. The collected voltage and current waveforms were processed into power metrics, i.e., real and reactive power time series as explained in section 3.1. In order to evaluate the performance of the NILM system, individual plug meters Enmetric Powerports, and ambient light sensors, i.e., Linksprite 
DiamondBack microcontrollers, equipped with a WiFi module and $\mathrm{AMBI}^{\mathrm{TM}}$ light intensity sensors, were used to provide the ground truth for plug loads and lighting fixtures, respectively. Prior to starting our experiment, the NILM system was first trained through a two-week real time training phase. During this period, at the time of using an appliance, the occupant was informed by the system's interface to manually label the detected event by choosing the proper appliance associated with that event. Following the training process, the NILM system automatically labeled the instances. Table 2 summarizes the possible events of activity-related appliances and the labels we used to represent them for our experiments. As demonstrated in table 2, the label of an event is a five-digit number with two parts separated by a zero. The first part is a three-digit number representing the appliance name. The second part is a one-digit number indicating the type of event (on or off). There are two types of events: "on" events with positive power change, represented by even numbers, and "off" events with negative power change, represented by odd numbers.

\begin{tabular}{|c|c|c|c|}
\hline Event & Label & Event & Label \\
\hline 11101 & Fridge light On & 20002 & Electric Grill Off \\
\hline 11102 & Fridge light Off & 18101 & Hair dryer On \\
\hline 16201 & Kettle/coffee maker On & 18102 & Hair dryer Off \\
\hline 16202 & Kettle/coffee maker Off & 16601 & Hair iron On \\
\hline 16301 & Toaster On & 16602 & Hair iron Off \\
\hline 16302 & Toaster Off & 12901 & Television On \\
\hline 16101 & Microwave On & 12902 & Television Off \\
\hline 16102 & Microwave Off & 12301 & Xbox On \\
\hline 20001 & Electric Grill On & 12302 & Xbox Off \\
\hline
\end{tabular}

The ground truth labels for activities were provided by a combination of occupants' written diaries, in which the occupants were asked to write down the start time and the duration of their performed activities as well as the appliances that were used during their activity, and annotations based on the sensor data playback (i.e., plug meters and light sensors) and interviewing occupants regarding their typical activities. The learned activities included: preparing breakfast, lunch, dinner and snack, watching television and grooming.

\subsection{Validation and discussion}

453
Following the completion of data collection, the input databases were first separated according to the separation categories identified via the algorithm presented in section 3.2. Along this line, for each unit, based on an occupant's daily activities and associated context information, i.e., an occupant's habits in performing the activities, appliances that are used during the activities and existing spaces in the unit, the TBox component of the knowledgebase for context-aware data separation was built. As explained in our proposed algorithm in section 3.2, by adding various ABox statements iteratively, the consistency of the knowledgebase under different scenarios of overlapping activities was tested. Using the algorithm, we detected the associated appliances with all possible overlapping activities perfectly. Based on these appliances, we determined the categories for data separation. These categories along with the characteristics of each test bed are provided in Table 3 .

\begin{tabular}{|c|c|c|c|c|}
\hline Test bed & $\begin{array}{c}\text { Test } \\
\text { duration } \\
\text { (days) }\end{array}$ & Spaces & Daily activities & $\begin{array}{c}\text { Categories of appliances for } \\
\text { data separation }\end{array}$ \\
\hline
\end{tabular}




\begin{tabular}{|c|c|c|c|c|}
\hline Unit 1 & 11 & $\begin{array}{l}\text { Kitchen, } \\
\text { Living } \\
\text { room, } \\
\text { Bedroom } \\
\quad \text { and } \\
\text { Bathroom }\end{array}$ & $\begin{array}{l}\text { Preparing Breakfast, } \\
\text { Preparing Lunch, } \\
\text { Preparing Dinner, } \\
\text { Preparing Snack, } \\
\text { Watching TV, } \\
\text { Grooming }\end{array}$ & $\begin{array}{c}\text { \{Microwave, Electric Kettle, } \\
\text { Fridge door, Toaster }\},\{T V, \\
\text { Hair dryer, Hair iron }\}\end{array}$ \\
\hline Unit 2 & 13 & $\begin{array}{l}\text { Kitchen, } \\
\text { Living } \\
\text { room, } \\
\text { Bedroom } \\
\quad \text { and } \\
\text { Bathroom } \\
\end{array}$ & $\begin{array}{l}\text { Preparing Breakfast, } \\
\text { Preparing Lunch, } \\
\text { Preparing Dinner, } \\
\text { Preparing Snack, } \\
\text { Watching TV, } \\
\text { Grooming }\end{array}$ & $\begin{array}{c}\{\text { Electric Kettle, Toaster }\},\{T V, \\
\text { Hair dryer }\}\end{array}$ \\
\hline Unit 3 & 16 & $\begin{array}{l}\text { Kitchen, } \\
\text { Bedroom } \\
\quad \text { and } \\
\text { Bathroom }\end{array}$ & $\begin{array}{l}\text { Preparing Breakfast, } \\
\text { Preparing Lunch, } \\
\text { Preparing Dinner, } \\
\text { Preparing Snack, } \\
\text { Watching TV }\end{array}$ & $\begin{array}{c}\{\text { Grill, Coffee maker Toaster }\} \\
\{T V, \text { Xbox }\}\end{array}$ \\
\hline
\end{tabular}

Table 3. Test beds characteristics and appliance categories for data separation identified using the method

The data that were separated based on the appliance categories were then used to detect the activity segments via the unsupervised segmentation process (explained in section 3.3.1). As noted before, datasets are analyzed on a 24-hour basis, i.e., a day. The starting point of days for each test bed was chosen in a way that no activity segment would be cut into two pieces. We chose these points by finding the common hours, in which the occupant has been inactive during the test days and therefore there was zero chance of cutting an activity segment. In Fig. 8, for each test bed, all occurred events during the test days are depicted. In addition, the selected starting point of days is shown by a vertical line. It can be seen that these points vary by case as different individuals have different daily routines. Since not everyone has consistent inactive periods through the days, finding a single starting point, which is applicable to all days, might not be possible. In these cases, days are first grouped based on their similarities and then each group is explored separately to find the starting point for segmentation. 


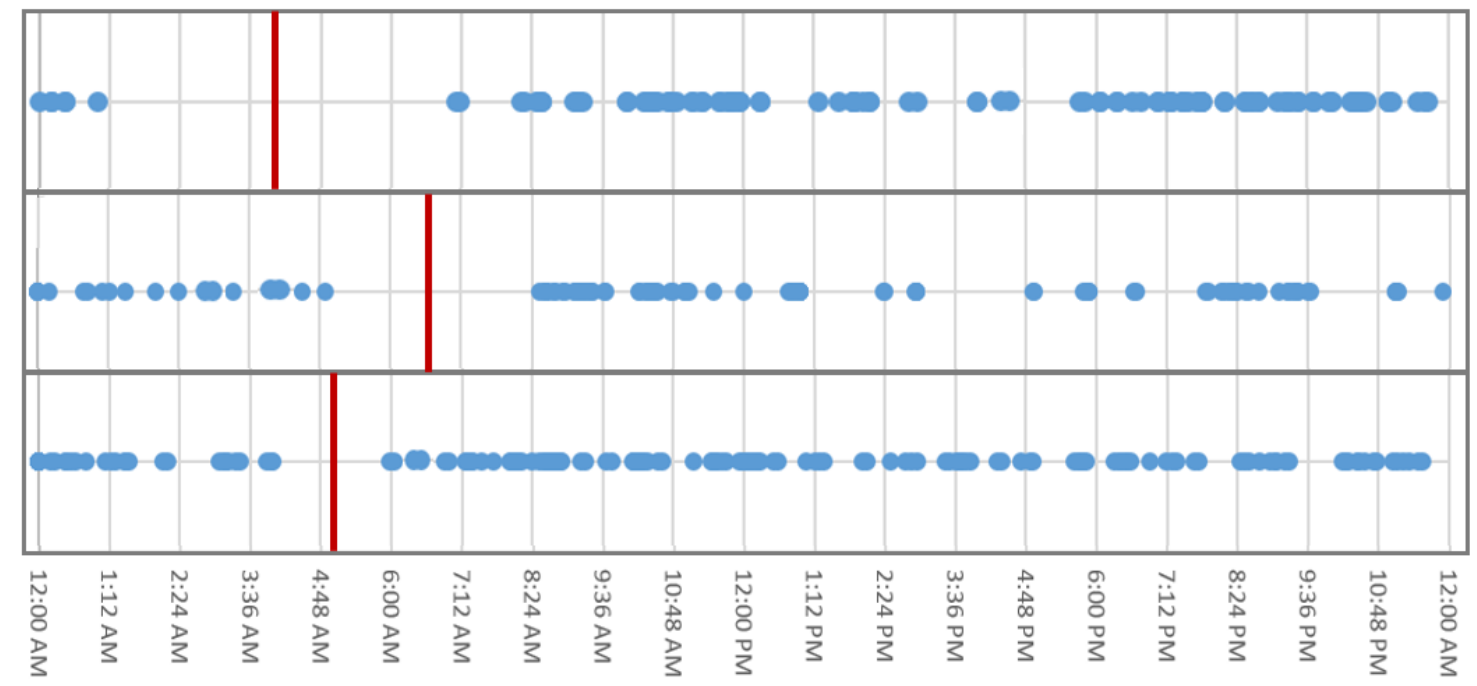

Fig. 8. Selected starting point of days for different test beds. The occurred events are shown in chronological order. The vertical red line shows the starting point of day.

In order to evaluate the segmentation results, the start time and duration of detected segments were compared with the ground truth duration and start time of the activities. The comparison results showed that the data was segmented with a precision of 0.95, a recall of 0.98 and a F-Measure value of 0.97 (Table 4). Since the precision is smaller than the recall, we concluded that the error was mainly a result from the true positives, which happens when there are gaps between groups of events within an activity segment.

\begin{tabular}{|c|c|c|c|c|}
\cline { 2 - 5 } \multicolumn{1}{c|}{} & $\begin{array}{c}\text { Number of Activity } \\
\text { Segments }\end{array}$ & Precision & Recall & F-Measure \\
\hline Unit 1 & 62 & 0.97 & 0.97 & 0.97 \\
\hline Unit 2 & 41 & 0.95 & 0.98 & 0.96 \\
\hline Unit 3 & 64 & 0.94 & 1.00 & 0.97 \\
\hline Total/Average & 167 & 0.95 & 0.98 & 0.97 \\
\hline
\end{tabular}

Following the segmentation, the feature vectors representing activity segments were computed. The feature vectors along with the associated ground truth labels were used to train selected common classification algorithms, including Random Forest, C4.5 Decision Tree, Naïve Bayes, and Support Vector Machine. In order to evaluate the performance of the stated algorithms, we carried out a 10 -fold cross validation. The average result of three units is summarized in Table 5.

\begin{tabular}{|c|c|c|c|c|}
\hline Classifier & Accuracy & Precision & Recall & F-Measure \\
\hline Random Forest & $93.41 \%$ & 0.934 & 0.934 & 0.931 \\
\hline C4.5 Decision Tree & $90.42 \%$ & 0.904 & 0.904 & 0.901 \\
\hline Naïve Bayes & $82.03 \%$ & 0.833 & 0.82 & 0.811 \\
\hline
\end{tabular}




\begin{tabular}{|l|l|l|l|l|} 
SVM & $86.83 \%$ & 0.874 & 0.868 & 0.861 \\
\hline
\end{tabular}

As depicted in Table 5, Random Forest classifier with average total accuracy of $93.41 \%$ outperformed the others. Hence, Random Forest was chosen as the classification technique to be used in our approach. The confusion matrix provided in Fig. 9 illustrates the average of the performance of the chosen classifier in classifying the instances corresponding to different classes of activities for three units.

\begin{tabular}{|c|c|c|c|c|c|c|}
\hline Classified as -> & $\mathrm{a}$ & $\mathrm{b}$ & $\mathrm{c}$ & $\mathrm{d}$ & $\mathrm{e}$ & $\mathrm{f}$ \\
\hline a: Preparing Breakfast & $\mathbf{9 4 . 2 9}$ & 5.71 & 0.00 & 0.00 & 0.00 & 0.00 \\
\hline b: Preparing Snack & 1.49 & $\mathbf{9 7 . 0 1}$ & 1.49 & 0.00 & 0.00 & 0.00 \\
\hline c: Preparing Dinner & 0.00 & 31.25 & $\mathbf{6 2 . 5 0}$ & 6.25 & 0.00 & 0.00 \\
\hline d: Preparing Lunch & 12.50 & 0.00 & 0.00 & $\mathbf{8 7 . 5 0}$ & 0.00 & 0.00 \\
\hline e: Watching Television & 0.00 & 0.00 & 0.00 & 0.00 & $\mathbf{1 0 0 . 0 0}$ & 0.00 \\
\hline f: Grooming & 0.00 & 0.00 & 0.00 & 0.00 & 0.00 & $\mathbf{1 0 0 . 0 0}$ \\
\hline
\end{tabular}

Table 6. Confusion matrix of the Random Forest classifier. The values show the percentage of instances in each class.

As shown in Fig. 9 the main misclassification of the classifier was related to the activities of preparing dinner and preparing lunch. There are two possible reasons for such a misclassification: 1) the short length of segments in some cases for preparing dinner, which has been resulted in a confusion with preparing snack; and 2) the close starting time of preparing lunch and preparing breakfast for some instances, which has led to misclassification into preparing breakfast.

The high average total accuracy achieved through our experimental validation, i.e., $93.41 \%$, is comparable with the accuracy of other high-performing activity recognition approaches. By relying on the data provided by a single sensing point, our proposed approach reduces the cost and complexity in comparison to common activity recognition approaches, which require installation of multiple sensors. Taking the work done by the authors in [21] and [37] as two examples of representative efforts in this area, in order to monitor an occupant's activities in a typical one bedroom apartment unit, there is a demand for at least 50 sensors, such as motion sensors, door sensors or ambient light sensors. Taking into consideration that the price of these types of sensors is about 10 dollars in average, 50 sensors would cost approximately 500 dollars. Moreover, in order to map the activities to energy consumption of appliances, there is a need for plug meters. This could cost at least an additional 500 dollars. Accordingly, in terms of the required sensors, using the existing activity recognition approaches, detection of daily activities and associating them with appliance usage would cost almost twice as much as what our proposed approach in this paper costs, i.e., 380 dollars for voltage and 160 dollars for current sensors. Moreover, to get 50 sensors working adequately along with the plug meters, they must be connected to one or more routers, which themselves are connected to a core computer with a software to manage and synchronize the data. This means using existing approaches for activity recognition also increases the costs associated with a wireless sensor network, such as the cost for the routers and also the software to manage and synchronize the data from various types of sensors with different sampling rates and data types. In addition, making a sensor network to function properly cannot be achieved by only providing these tools, as sensor failure and loss of connection are known drawbacks of wireless sensor networks. Hence, in order to address these drawbacks, there is a need for network auditing by an expert on a regular basis. Due to the high number of sensing points, which are connected wirelessly 
to the main computer and which continuously send data, the labor commitment and consequently the cost associated with auditing the sensor network system is much higher than the cost of auditing our system, in which there is only one sensing point that is connected to the main computer with wires. Based on this discussion, the difference between the cost and complexity of existing approached and cost and complexity of our approach, is much higher than simply the difference between the costs of the required sensors, as our approach reduces the cost and complexity by eliminating the need for a wireless network and dedicated labor.

As stated in section 2, achieving high accuracies in activity recognition requires the implementation of supervised learning algorithms. Consequently, user engagement during the training process, in which the user records the activities along with activity start and end times is a common requirement in all highperforming daily activity recognition approaches. Therefore, compared to the existing work, e.g., [21] and [37], in terms of type of user engagement our approach is no exception, as it does not add any extra burden on the user during the training period. Considering the presented results in three test beds, with average training period of 13 days, our approach is also similar to the existing approaches, in terms of the required time for training of the activity recognition model. However, what is different in our case is that in addition to the training requirement for activity recognition, there is a necessity for training of the NILM system, which, after all, results in user engagement over a longer period of time. Yet, we can potentially combine these two efforts, which is part of our planned future work.

Following the activity detection, the approximate electricity consumption of the detected activities was estimated, based on the average power consumption and the usage duration of appliances within the corresponding activity segments. As the main focus of this paper is the activity recognition, we did not investigate the precise calculation of energy consumption of the appliances. Accordingly, in order to achieve an acceptable approximation, we compared our calculated power consumption of appliances with average power consumption from the plug meters and for cases where the difference was more than $10 \%$, we used the plug meter value. Fig. 10 presents the average contribution of different appliances in electricity consumption associated with daily activities in the three units during the experiment period. In addition to the contribution of appliances in activities, we also explored the total electricity consumption of each activity by adding up the electricity consumption of associated appliances during an activity. In table 6 , the average daily total electricity consumption (in $\mathrm{kWh}$ ) of investigated activities in this experiment, are presented for three units.

\begin{tabular}{|c|c|c|c|}
\hline \multirow{2}{*}{ Daily Activity } & \multicolumn{3}{|c|}{ Average daily electricity consumption $(\mathrm{kWh})$} \\
\cline { 2 - 4 } & Unit 1 & Unit 2 & Unit 3 \\
\hline Watching TV & 0.194051 & 1.057254 & 2.189193 \\
\hline Preparing Dinner & 0.148056 & 0.126499 & 4.926559 \\
\hline Preparing Lunch & 0.129812 & 0.041424 & 0.445365 \\
\hline Preparing Breakfast & 0.129404 & 0.236948 & 0.358391 \\
\hline Grooming & 0.057007 & 0.061828 & - \\
\hline Preparing Snack & 0.00698 & 0.047295 & 0.163462 \\
\hline
\end{tabular}




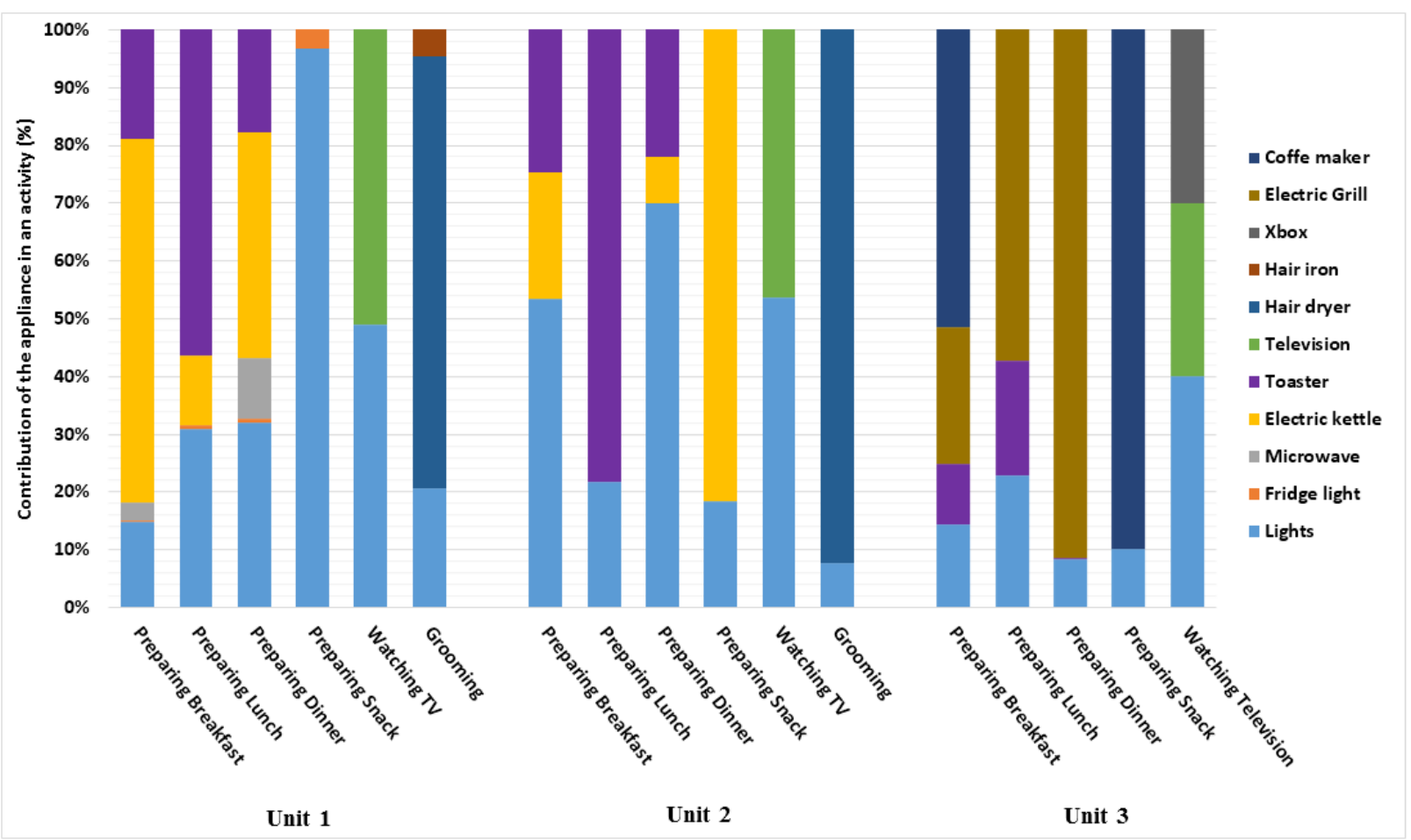

Fig. 9. Contribution of appliances in electricity consumption of daily activities for three units

Based on the presented bar chart and table, following insights regarding occupant's behavior in tested units can be achieved:

Activity of preparing meals: The first three bars for each unit in Fig. 10 represent the activity of preparing meals in different times of the day (breakfast, lunch and dinner). It can be seen from the chart that although they are representing the same type of activity (preparing meals), the contributions of appliances are not similar in all three cases. In unit 1 , while the electric kettle has the highest contribution in consuming electricity for preparing breakfast, the toaster plays this role for preparing lunch in this unit. Although the contribution of toaster in preparing dinner and preparing breakfast is similar, due to an increase in microwave usage, it can be seen that the contribution of the electric kettle for preparing dinner is $20 \%$ less than the one for preparing breakfast. In unit 2, the contribution of toaster follows the same pattern as unit 1 , which has similar values for preparing breakfast and preparing dinner and a significantly higher value (higher than all other contributors) for preparing lunch. On the other hand, unlike unit 1, for preparing breakfast and dinner in unit 2, lights play the role of the highest contributor to electricity consumption. In unit 3, except for preparing breakfast, in which the coffee maker has the highest contribution, the main consumption is associated with the electric grill. The frequent use of the electric grill by the occupant in this unit is the main reason behind the high electricity consumption of the meal preparing activities (preparing breakfast, lunch and dinner), compared to other units (Table 6). By less frequent use of the electric grill or switching to a more efficient one, this occupant can considerably reduce the energy consumption of this activity.

Activity of preparing snack: The fourth bar in Fig. 10 shows the activity of preparing snack. As illustrated, the occupants of unit 2 and unit 3 have similar routines for snacking, as they both use the appliances for preparing hot drinks, i.e., electric kettle in unit 2 and coffee maker in unit 3 . The occupant of unit 1 , on the other hand, does not desires hot drinks for snacking, as the fridge light, which is sign for taking food out of fridge, is the only source of electricity consumption in addition to the kitchen lights in this unit. Accordingly, the energy consumption of preparing snack in unit 1 is significantly lower than other units. 
Activity of grooming: As shown in in Fig. 10, for activity of grooming, there is $13 \%$ difference between the contribution of lights in unit 1 and the one in unit 2. Since the total electricity consumption of this activity is only $0.08 \%$ higher in unit 2 , it can be concluded that in unit 1 , the lights consume more electricity during the activity of grooming compared to the one in unit 2 .

Activity of watching television: Watching television is the most electricity consuming activity in unit 1 and unit 2 and the second most electricity consuming activity in unit 3. However, as illustrated in Table 6, there is a large difference in total electricity consumption value of this activity among different units (it is 5 and 11 times bigger than unit 1 in unit 3 and unit 2, respectively). The stated variation is mainly due to the difference in hours that an occupant spends on this activity. Accordingly, it can be concluded that the occupants of unit 2 and unit 3 spend much more hours on watching television compared to the occupant in unit 1 does. Based on the bar chart, for activity of watching television in all units, the lights contribute to almost the same portion of electricity consumption as other associated appliances (television for unit 1 and unit 2 and combination of Xbox and television for unit 3) do. Typically while watching television, one does not need a highly bright environment. Hence, by dimming down the high energy consuming lights, there is a potential to reduce the electricity consumption during this activity. As stated before, since watching television is among the highest electricity consumption activity in all units, the explained saving strategy could lead to a considerable amount of savings in a year.

The provided comparison of the activities among different units showed how behaviors might vary by occupants and also revealed the potentials for energy saving. While achieving some of these potentials requires behavior change, there are energy saving potentials that can be achieved by providing automation in the building. For example, as explained in the results, dimming down the lights during the activity of watching television can potentially save considerable amounts of energy in long term. Here, energy could be saved either by modifying occupant behavior through changing the light level manually during this activity or by adding an automation system, which dims down the light level when the activity of watching television is recognized.

\section{Limitations and future work}

In this study, we investigated the application of activity recognition to get insights about occupant behaviors in consuming electricity during the performance of daily activities. The objective of this paper is to propose a framework to allocate appliance-level disaggregated electricity consumption to daily activities using a single sensing point. We carried out experiments in three single occupancy test bed apartment units, in order to evaluate our proposed framework rather than generalizing the discovered knowledge from the three cases to all population. The high accuracy achieved through our experimental validation for activity recognition, demonstrated the validity of our proposed activity recognition approach for single occupancy apartment units. However, there are limitations associated with our study that we will explore in our future work. First, in this study, our validation was limited to five groups of activities. Other activities will be explored in our future works. Second, in our presented framework, the active segments are detected based on the assumption that the activities are associated with the segments with relatively dense events. However, the correctness of this assumption is still under question when more complex activities are investigated or activities where multiple occupants are involved (e.g., social activities). Another limitation of our framework is the performance of our ontology-based algorithm in detecting accurate groups of appliances for data separation. Since we were investigating a small number of activities in single-occupancy test beds, modeling of the context information and accordingly construction of the ontologies were not complicated tasks and the results were satisfying. However, our proposed algorithm must be tested for more complicated cases with more context variations. Finally, in this study, we were using single source of input data, i.e., disaggregated appliance usage; therefore, we could obtain a certain level of insight about occupant's activities. By using supplementary information, such as occupant's location, it is possible to gain deeper insights and consequently provide the occupant with more useful energy saving recommendations. Based 
on the stated limitations, in our future studies, we will explore the generalizability of our framework by investigating more complicated cases, such as test beds with multiple occupants or test beds with occupants from different demographics. Since in this study our main focus was the recognition of the daily activities using a single sensing point, we used an approximate approach to estimate energy consumption of appliances. In future, we will investigate and evaluate more precise approaches of estimating the energy consumption. Although, in this study, we used two separate training procedures for the NILM system and the activity recognition, in our future studies, we will investigate the potential of combining these two training procedures in order to reduce the burden on users as much as possible. In addition, we will study the use of supplementary information, such as occupant locations, to investigate broader ways of saving energy by getting deeper insights about different behaviors and preferences of occupants and also the effects of their activities and behaviors on background appliances in buildings.

\section{Conclusions}

In this paper, we presented a framework to allocate appliance-level electricity consumption to daily activities of occupants via activity recognition. Our presented framework consists of three main parts: context-aware data separation, segmentation and activity recognition, and electricity consumption estimation. The input of this framework is the disaggregated appliance usage data provided by a NILM system. In order to separate overlapping activities, we introduced an ontology-based approach, based on which the input data are separated into categories with regards to the context information. Then the separated data are segmented into active and inactive segments. Next, the active segments are mapped into activities. Finally, the associated electricity consumption of detected activities is estimated. In order to evaluate our framework, an experimental validation in three single occupancy apartment units was carried out. The experimental results showed a total F-measure value of 0.97 for segmentation and an average accuracy of $93.41 \%$ for activity recognition. Even though in our proposed framework we used a single sensing point, the high accuracy achieved through our experimental validation is comparable with the accuracy of other high-performing activity recognition approaches, which commonly requires installation of multiple sensors. Following the detection of activities, the approximate electricity consumption associated with each activity was estimated. The differences among contribution of appliances in electricity consumption of investigated activities provided us with insights about different behaviors in performing daily activities in tested units. These insights might be further used to give energy saving recommendations to occupants.

\section{Acknowledgements}

This material is based upon work supported by the National Science Foundation under Grants \# 1231001 and 0930868. Any opinions, findings, and conclusions or recommendations expressed in this material are those of the authors and do not necessarily reflect the views of the National Science Foundation.

\section{References}

[1] Annual Energy Outlook U.S. Energy Information Administration, (2015).

[2] T.A. Nguyen, M. Aiello, Energy intelligent buildings based on user activity: A survey, Energy and buildings, 56 (2013) 244-257.

[3] L. Farinaccio, R. Zmeureanu, Using a pattern recognition approach to disaggregate the total electricity consumption in a house into the major end-uses, Energy and Buildings, 30 (3) (1999) 245-259.

[4] J. Froehlich, E. Larson, S. Gupta, G. Cohn, M.S. Reynolds, S.N. Patel, Disaggregated end-use energy sensing for the smart grid, IEEE Pervasive Computing, (1) (2010) 28-39.

[5] F. Jazizadeh, S. Ahmadi-Karvigh, B. Becerik-Gerber, L. Soibelman, Spatiotemporal lighting load disaggregation using light intensity signal, Energy and Buildings, 69 (2014) 572-583. 
[6] J. Paauw, B. Roossien, M. Aries, O.G. Santin, Energy Pattern Generator; Understanding the effect of user behaviour on energy systems, in: First European Conference Energy Efficiency and Behaviour, 2009.

[7] J. Fechner, Human factors in appliance energy-consumption, in: Proceedings of the IEEE Appliance Technical Conference, Pittsburgh, Pennsylvania, 1977.

[8] WBCSD, Transforming the Market: Energy Efficiency in Buildings, Survey report, The World Business Council for Sustainable Development, (2009).

[9] G.W. Hart, Nonintrusive appliance load monitoring, Proceedings of the IEEE, 80 (12) (1992) 18701891.

[10] A. Avci, S. Bosch, M. Marin-Perianu, R. Marin-Perianu, P. Havinga, Activity recognition using inertial sensing for healthcare, wellbeing and sports applications: A survey, in: Architecture of computing systems (ARCS), 2010 23rd international conference on, VDE, 2010, pp. 1-10.

[11] V. Osmani, S. Balasubramaniam, D. Botvich, Self-organising object networks using context zones for distributed activity recognition, in: Proceedings of the ICST 2nd international conference on Body area networks, ICST (Institute for Computer Sciences, Social-Informatics and Telecommunications Engineering), 2007, pp. 14.

[12] A. Serna, H. Pigot, V. Rialle, Modeling the progression of Alzheimer's disease for cognitive assistance in smart homes, User Modeling and User-Adapted Interaction, 17 (4) (2007) 415-438. [13] M. Berenguer, M. Giordani, F. Giraud-By, N. Noury, Automatic detection of activities of daily living from detecting and classifying electrical events on the residential power line, in: e-health Networking, Applications and Services, 2008. HealthCom 2008. 10th International Conference on, IEEE, 2008, pp. 29-32.

[14] M. Mubashir, L. Shao, L. Seed, A survey on fall detection: Principles and approaches, Neurocomputing, 100 (2013) 144-152.

[15] J.M. Abreu, F.C. Pereira, P. Ferrão, Using pattern recognition to identify habitual behavior in residential electricity consumption, Energy and buildings, 49 (2012) 479-487.

[16] Z. Yu, B.C. Fung, F. Haghighat, H. Yoshino, E. Morofsky, A systematic procedure to study the influence of occupant behavior on building energy consumption, Energy and Buildings, 43 (6) (2011) 1409-1417.

[17] J. Virote, R. Neves-Silva, Stochastic models for building energy prediction based on occupant behavior assessment, Energy and Buildings, 53 (2012) 183-193.

[18] I. Richardson, M. Thomson, D. Infield, C. Clifford, Domestic electricity use: A high-resolution energy demand model, Energy and Buildings, 42 (10) (2010) 1878-1887.

[19] J. Widén, M. Lundh, I. Vassileva, E. Dahlquist, K. Ellegård, E. Wäckelgård, Constructing load profiles for household electricity and hot water from time-use data-Modelling approach and validation, Energy and Buildings, 41 (7) (2009) 753-768.

[20] D. Bourgeois, C. Reinhart, I. Macdonald, Adding advanced behavioural models in whole building energy simulation: a study on the total energy impact of manual and automated lighting control, Energy and Buildings, 38 (7) (2006) 814-823.

[21] B.L. Thomas, D.J. Cook, CARL: activity-aware automation for energy efficiency, in: Proceedings of the 2014 ACM International Joint Conference on Pervasive and Ubiquitous Computing: Adjunct Publication, ACM, 2014, pp. 939-946.

[22] S. Lee, G. Ryu, Y. Chon, R. Ha, H. Cha, Automatic Standby Power Management Using Usage Profiling and Prediction, Human-Machine Systems, IEEE Transactions on, 43 (6) (2013) 535-546. [23] C. Chen, D.J. Cook, A.S. Crandall, The user side of sustainability: Modeling behavior and energy usage in the home, Pervasive and Mobile Computing, 9 (1) (2013) 161-175.

[24] N.D. Rodríguez, M.P. Cuéllar, J. Lilius, M.D. Calvo-Flores, A survey on ontologies for human behavior recognition, ACM Computing Surveys (CSUR), 46 (4) (2014) 43.

[25] P. Rashidi, D.J. Cook, Keeping the resident in the loop: Adapting the smart home to the user, Systems, Man and Cybernetics, Part A: Systems and Humans, IEEE Transactions on, 39 (5) (2009) 949959. 
[26] F. Li, S. Dustdar, Incorporating Unsupervised Learning in Activity Recognition, in: Activity Context Representation, 2011.

[27] T. Van Kasteren, A. Noulas, G. Englebienne, B. Kröse, Accurate activity recognition in a home setting, in: Proceedings of the 10th international conference on Ubiquitous computing, ACM, 2008, pp. $1-9$.

[28] T.V. Duong, H.H. Bui, D.Q. Phung, S. Venkatesh, Activity recognition and abnormality detection with the switching hidden semi-markov model, in: Computer Vision and Pattern Recognition, 2005. CVPR 2005. IEEE Computer Society Conference on, IEEE, 2005, pp. 838-845.

[29] D. Anguita, A. Ghio, L. Oneto, X. Parra, J.L. Reyes-Ortiz, Human activity recognition on smartphones using a multiclass hardware-friendly support vector machine, in: Ambient assisted living and home care, Springer, 2012, pp. 216-223.

[30] Y. Du, F. Chen, W. Xu, Y. Li, Recognizing interaction activities using dynamic bayesian network, in: Pattern Recognition, 2006. ICPR 2006. 18th International Conference on, IEEE, 2006, pp. 618-621. [31] B. Logan, J. Healey, M. Philipose, E.M. Tapia, S. Intille, A long-term evaluation of sensing modalities for activity recognition, Springer, 2007.

[32] L. Bao, S.S. Intille, Activity recognition from user-annotated acceleration data, in: Pervasive computing, Springer, 2004, pp. 1-17.

[33] D. Riboni, C. Bettini, OWL 2 modeling and reasoning with complex human activities, Pervasive and Mobile Computing, 7 (3) (2011) 379-395.

[34] L. Chen, C. Nugent, Ontology-based activity recognition in intelligent pervasive environments, International Journal of Web Information Systems, 5 (4) (2009) 410-430.

[35] R. Bodor, B. Jackson, N. Papanikolopoulos, Vision-based human tracking and activity recognition, in: Proc. of the 11th Mediterranean Conf. on Control and Automation, Citeseer, 2003.

[36] D. Weinland, R. Ronfard, E. Boyer, A survey of vision-based methods for action representation, segmentation and recognition, Computer Vision and Image Understanding, 115 (2) (2011) 224-241. [37] E.M. Tapia, S.S. Intille, K. Larson, Activity recognition in the home using simple and ubiquitous sensors, Springer, 2004.

[38] M. Stikic, T. Huynh, K.V. Laerhoven, B. Schiele, ADL recognition based on the combination of RFID and accelerometer sensing, in: Pervasive Computing Technologies for Healthcare, 2008. PervasiveHealth 2008. Second International Conference on, IEEE, 2008, pp. 258-263. [39] D. Luo, L.K. Norford, S.R. Shaw, S.B. Leeb, Monitoring HVAC equipment electrical loads from a centralized location--methods and field test results/Discussion, ASHRAE Transactions, 108 (2002) 841. [40] M. Berges, E. Goldman, H.S. Matthews, L. Soibelman, K. Anderson, User-centered nonintrusive electricity load monitoring for residential buildings, Journal of Computing in Civil Engineering, 25 (6) (2011) 471-480.

[41] S.R. Shaw, S.B. Leeb, L.K. Norford, R.W. Cox, Nonintrusive load monitoring and diagnostics in power systems, Instrumentation and Measurement, IEEE Transactions on, 57 (7) (2008) 1445-1454. [42] F. Jazizadeh, B. Becerik-Gerber, M. Berges, L. Soibelman, An unsupervised hierarchical clustering based heuristic algorithm for facilitated training of electricity consumption disaggregation systems, Advanced Engineering Informatics, 28 (4) (2014) 311-326.

[43] S. Staab, R. Studer, Handbook on ontologies, Springer Science \& Business Media, 2013.

[44] E. Parzen, On estimation of a probability density function and mode, The annals of mathematical statistics, (1962) 1065-1076.

[45] H. Shimazaki, S. Shinomoto, Kernel bandwidth optimization in spike rate estimation, Journal of computational neuroscience, 29 (1-2) (2010) 171-182. 